\title{
Epidemiologia e Saúde Coletiva: tendências da produção epidemiológica brasileira quanto ao volume, indexação eáreas de investigação - 2001 a 2006
}

\author{
Epidemiology and Public Health: \\ tendencies of the Brazilian epidemiology production \\ regarding volume, indexation and investigation areas - 2001 a 2006
}

Silvana Rubano Barretto Turci ${ }^{1}$

M aria Cristina Rodrigues Guilam ${ }^{1}$

MariaClara Coelho Câmara ${ }^{1}$

${ }^{1}$ Centro de Estudos da Saúdedo Trabalhador e Ecologia Humana, Escola Nacional deSaúdePública, Fundação O swaldo Cruz. Rua Leopoldo Bulhões 1480 , M anguinhos. 21041-210 Rio de Janeiro RJ.srubano@ensp.fiocruz.br
Abstract Thisarticle examines and interprets the trends of epidemiological production in Brazil in the Public $\mathrm{H}$ ealth context. CAPES indicators from 2001 to 2006 were used as database. We analyzed 26 programs and select the 10 major ones to analyzetheir bibliographic production in indexed journals. It was observed that the total production of epidemiological articles accounted for $40 \%$ of production in Public $\mathrm{Health}$ and $55 \%$ of the production was published in international journals, especially Cadernos de Saúde Pública, Revista de SaúdePública and Ciência \& SaúdeColetiva. The most prevalent themes were public health nutrition, maternal and infant health and, infectious diseases, particularly AIDS. Environmental e worker's heath, oral health, violence and health of the elderly have been ratified as objects of study for thediscipline, whilemeta-analysis and geoprocessing appear as a useful tool for health services. We conclude that the epidemiological production tends to increase in indexed publications, covering various topics and a wide spectrum of relevant issues to Brazilian health policy.

Key words Epidemiology, Epidemiologic research, Public health
Resumo 0 presente artigo examina einterpreta as tendências da produção epidemiológica no Brasil, no contexto da Saúde Coletiva. U tilizou-se, como banco dedados, os Cadernos de Indicadores da Capes, no período de 2001 a 2006. Foram analisados 26 programas, sendo que os dez maiores tiveram sua produção bibliográfica em periódicosindexados examinada. O bservou-sequea produção total de artigos epidemiológicos representou $40 \%$ da produção em Saúde Coletiva e 55\% da produção em revistas internacionais, destacando-se Cadernos de Saúde Pública, Revista de Saúde Pública e Ciência \& Saúde Coletiva. Os temas mais prevalentes foram nutrição em saúde pública, saúde materno-infantil e doenças infectocontagiosas, particularmentea aids. Saúde-trabalho-ambiente, saúde oral, violência e saúde do idoso vêm se consolidando como objeto de estudo da disci plina, enquanto estudos de meta-análisee geoprocessamento aparecem como ferramenta útil para os serviços de saúde. Conclui-se que há tendência de aumento da produção epidemiológica em publicações indexadas, abrangendo temáticas diversas e abarcando amplo espectro de aspectos relevantes para a política de saúde no país. Palavras-chave Epidemiologia, Pesquisa epidemiológica, Saúde coletiva 
Introdução

0 presente artigo tem por objetivo analisar o desenvolvimento da pesquisa epidemiológica no Brasil, abordando aspectos históricos, institucionais e tendências atuais no contexto da Saúde Coletiva. Este estudo é uma das etapas do projeto "Avaliação da pós-graduação na área de Saúde Coletiva sob as perspectivas dos atores, da cultura, das tendências e das várias formas de expressão acadêmica", desenvolvido pela Associação Brasileira de Saúde Coletiva (Abrasco).

Embora não haja consenso em sua definição, a epidemiologia é entendida, em sentido amplo, como o estudo do comportamento da doença em populações ${ }^{1}$. No Brasil, seu ensino teve início nos cursos de medicina nos anos vinte enos anos setenta foi incorporado a cursos de pós-graduação ${ }^{2}$. Já na década de oitenta, a disciplina sofreu forteinfluência dostrabalhos deAsa Cristina Laurel e Juan Cesar Garcia, incorporando a discussão da relação entreclassesocial esaúde, além das idéias de M ilton Santos sobregeografia crítica que influenciaram os estudos de endemias, através da contribuição de Carvalheiro ${ }^{3}$. Destaca-se, ainda, a importância histórica dos estudos sobre causalidade desenvolvidos por Arouca ${ }^{4}$ e Silva, 6 .

Pode-se observar a influência de distintas correntes de pensamento na constituição da epidemiologia brasileira, que se mantém, no entanto, sempre associada à Saúde Pública. Barreto ${ }^{7}$ observa que o contexto nacional possibilitou a emergência de um conjunto de novos autores "híbridos de epidemiólogos-epistemólogos", como Almeida Filho et al. ${ }^{8}$, Ayres ${ }^{9}$, Castiel et al. ${ }^{10}$ eCzeresnia ${ }^{11,12}$, cuja produção se volta para a reflexão sobre a disciplina e suas relações com outras ciências e campos de práticas.

Por agregar o arcabouço teórico-metodológico à prática dos serviços de saúde, pode-se dizer que a epidemiologia tem dupla face: por um lado, produz novos conhecimentose, por outro, colabora para efetivas transformações nas condições de vida e saúde das populações ${ }^{13}$. Do ponto de vista das políticas públicas, contribui para o planejamento, gestão e avaliação de ações e programas de saúde pública, através da proposição de medidas para o controle e/ou minimização de agravos. Portanto, os estudos epidemiológicos são úteis para a descrição das condições de saúde de uma população selecionada, considerando-se o tempo e o espaço; para investigar fatores determinantes da saúde da população; para avaliar o impacto das ações efetuadas com o objetivo de alterar uma dada situação de saúde eestabelecer uma relação de associação entreum suposto fator de risco e o desfecho, que pode ou não ser a doença.

No que concerne o desenvolvimento da epidemiologia no Brasil, Barreto ${ }^{7}$ identifica dois marcos históricos fundamentais: a I Reunião Nacional sobre Ensino e Pesquisa em Epidemiologia, realizada em 1984, e o encontro intitulado "Rumos da Epidemiologia Brasileira - reunião nacional de avaliação e perspectivas", realizado em 1994.

$\mathrm{Na}$ I Reunião Nacional sobre Ensino e Pesquisa em Epidemiologia, realizada em N ova Friburgo (RJ), foi criada a Comissão de Epidemiologia da Abrasco ${ }^{14}$. Essa comissão, além de selar um compromisso para a melhoria da saúde pública, estreitou os laços entre os epidemiologistas da academia e dos serviços de saúde, através da promoção de eventos científicos, reuniões e seminários, e facilitou a publicação e divulgação dos conhecimentos produzidos. A criação da Revista Brasileira deEpidemiologia em 1998 éum dos exemplos da atuação dessa comissão. Além disso, participou ativamente da elaboração e coordenação deuma política de ciência etecnologia para a Saúde Coletiva e formulou planos diretores para o desenvolvimento da epidemiologia no país, contemplando questões de ensino, pesquisa e práticas em serviços de saúde. Segundo Barreto" , a comissão é "a grande tecelã deste tecido complexo que é a epidemiologia no Brasil".

0 relatório final da reunião, divulgado em 1986, propõe uma nova visão da epidemiologia que incorpora aspectos sociais aos métodos da disciplina e sua aplicação no planejamento de serviços de saúde.

O segundo marco histórico apontado por Barreto ${ }^{7}$, e também promovido pela comissão de epidemiologia da Abrasco, foi o encontro "Rumos da Epidemiologia Brasileira: reuniões nacionais de avaliação e perspectivas", em Olinda $(1994)^{15}$. Os participantes desse encontro assinalaram queo processo de consolidação do Sistema Único de Saúde (SUS) estava modificando o papel da epidemiologia no Brasil. Uma abordagem globalizante das condições de saúde edo perfil de adoecimento dos grupos populacionais foi proposta, em contraposição ao enfoque tradicional fragmentado esetorizado da vigilância epidemiológica de algumas enfermidades transmissíveis.

0 evento apontou, ainda, para o papel relevantedo Centro Nacional deEpidemiologia (CENEPI), na época, em fase de consolidação. Tal centro passaria a atuar como órgão central, coordenador das ações de epidemiologia no sistema 
nacional de saúde do país e estimulador da formação de áreas de vigilância epidemiológica nos níveis estadual emunicipal, o queimplicaria uma maior descentralização das ações. Criado em 1990, - CENEPI originou-se com a reforma sanitária, movimento iniciado na década de oitenta. Teve como missão promover o uso da epidemiologia em todos os níveis do SUS, buscando subsidiar a formulação e a implementação das políticas de saúde nacionais. Esse processo desenvolveu uma longa tradição de trabalho conjunto, integrando universidades e serviços de saúde para o estabelecimento e consolidação de sistemas de informação, capacitação de recursos humanos e apoio à pesquisa estratégica. Além disso, o CENEPI acumulou experiência em vigilância epidemiológica de agravos e doenças, papel hoje desempenhado pela Secretaria de Vigilância em Saúde.

Cabe destacar que a atuação da $\mathrm{Abrasco}^{15}$ também foi importante nos anos de 1986 e 1989 , quando promoveu dois eventos de relevância histórica para a epidemiologia. 0 primeiro foi 0 Seminário sobre Perspectivas da Epidemiologia frente à Reorganização dos Serviços de Saúde, em Itaparica (BA), cujo relatório evidencia a preocupação dos epidemiologistas com a formação de profissionais capazes deintervir concretamente no sistema de saúde.

O segundo evento, "Estratégias para o desenvolvimento da epidemiologia no Brasil", resultou na formulação do I Plano Diretor quepreconizou o primeiro pacto político formal para 0 desenvolvimento da epidemiologia no país. Esse pacto envolveu instituições acadêmicas, de fomento científico e de serviços de saúde ${ }^{15}$.

Além da Abrasco, instituições como a O rganização Pan-Americana da Saúde(OPAS) ea Fundação Oswal do Cruz, através da Escola N acional deSaúde Pública (ENSP), desempenharam papel fundamental para o desenvolvimento da epidemiologia no país, bem como para sua aproximação com os serviços de saúde. Tal aproximação pode ser identificada através de diversas contribuições que a área da Saúde Coletiva tem dado para a gradativa redução da mortalidade infantil, aumento da expectativa de vida e a diminuição/ eliminação de várias doenças infecciosas. Essas conquistas foram possíveis por que houve maior capacidade de gestão, aumento do número de profissionais qualificados e um elenco de investigadores dedicados, fruto dessa interrelação positivaentrea epidemiologia eaSaúdeColetiva. Cabe ressaltar que esses avanços envolvem também outros aspectos, não apenas técnicos nem somente relacionados ao setor saúde.
Com relação à produção acadêmica no Brasil, a epidemiologia tem uma história rica e recente. Esta riqueza pode ser constatada através do aumento vertiginoso dos grupos de pesquisas cadastrados no Diretório de Pesquisas do CNPq, diversidade de linhas temáticas, bem como um expressivo aumento do número de artigos publicados em revistas indexadas ${ }^{16}$.

Silva ${ }^{5,6}$ destaca que a produção do conhecimento epidemiológico no Brasil oscilou, nos seus primórdios, entre o rural e o urbano. Nos primeiros cinquenta anos do século $X X$, a produção acadêmica voltou-se predominantemente para o controle das doenças endêmicas rurais, como febre amarela e doença de Chagas. Em seguida, é possível verificar um deslocamento para produção de estudos sobre o controle de doenças endêmicas urbanas, como hanseníase, tuberculose e meningite. O bserva-se hoje um conjunto de pesquisas que refletem a coexistência de doenças transmissíveis, que nunca chegaram a ser efetivamente controladas, como a tuberculose e a malária, com doenças emergentes como a aids, além do ressurgimento de doenças como a denguee o crescimento das doenças crônicas que eram características de países desenvolvidos. Essa superposição de eventos implicauma concentração de carga de doenças que configura um perfil particular de morbimortalidade, altamente desafiante para a saúde pública brasileira ${ }^{17}$.

Neste sentido, cabe aos epidemiologistas e à epidemiologia, aliado aos enfoques do planejamento e das ciências sociais, a grande tarefa de reorganização do setor saúde, buscando a integração entre a academia e os serviços de saúde pública.

Diante da importância que a epidemiologia tem para a área de saúde pública e de seu crescimento enquanto disciplina nos diversos programas de graduação e pós-graduação, buscamos analisar a produção acadêmica a partir das linhas de pesquisa e das publicações em revistas indexadas. Partimos do pressuposto que a epidemiologia contribui com grande parte da produção da área e que vem debatendo temas tradicionais e incorporando novos desafios para a saúde pública.

\section{M étodo}

Para concretização deste estudo, inicialmente foram identificadas as bases de dados da Coordenação de Aperfeiçoamento de Pessoal de Nível Superior (CAPES) disponíveis no portal ${ }^{18}$. Foram 
identificadas as instituições deSaúdeColetiva que oferecem cursos de pós-graduação stricto sensu mestrado acadêmico e doutorado. A seguir, foram selecionados os programas que oferecem formação em epidemiologia, seja como subárea deconhecimento, linha de pesquisa ou como programa específico. A partir dessa seleção, realizouse um levantamento nos cadernos de indicadores daCAPES para analisar a produção científica destes programas no período de 2001 a 2006 (período de realização do projeto mencionado na introdução). 0 levantamento privilegiou as seguintes informações: total de programas da área de Saúde Coletiva; linhas de pesquisa dos programas; produção de artigos publicados em revistas indexadas. Inicialmente, foram analisados os 26 programas que disponibilizaram cadernos de indicadores no período da pesquisa. Para a análise de artigos, especificamente, foram considerados os dez programas que apresentaram maior produção em periódicos indexados, em números absolutos; a saber, Programa de Saúde Pública da Escola Nacional de Saúde Publica da Fiocruz (ENSP-Fiocruz), Programa de Saúde Pública da Faculdade de Saúde Pública da Universidade de São Paulo (SP-USP), Programa de M edicina Preventiva da Faculdade de M edicina da Universidade de São Paulo (M P-USP), Programa de Epidemiologia da Universidade Federal do Rio Grande do Sul (UFRGS), Programa de Epidemiologia da Universidade Federal de Pelotas (UFPEL), Programa de Saúde Coletiva do Instituto de Saúde Coletiva da Universidade Federal da Bahia (ISCBA), Programa de Saúde Coletiva do Instituto de M edicina Social da Universidade Estadual do Rio de Janeiro (IMS-UERJ), Programa de Saúde Coletiva da Universidade Estadual de Campinas (U NICAM P), Programa deSaúdePública da Universidade Federal deM inas Gerais (UFM G) ePrograma de Saúde Coletiva do Instituto de Estudos em Saúde Coletiva da Universidade Federal do Rio de Janeiro (IESC-UFRJ).

Para definir artigo epidemiológico, foram realizadas buscas nos campos: título, resumo e palavras-chave. Os argumentos de busca utilizados constituíram termos selecionados do vocabulário DeCS (http://decs.bvs.br/cgi-bin/ wxis1660.exe/decsserver/), nas subcategorias SP3 eSP ${ }^{19}$. Foram incluídos os seguintes descritores: epidemiologia; epidemiologia descritiva; epidemiologia analítica; métodos epidemiológicos; estudos epidemiológicos (caso-controle, coorte, transversais, ensaios clínicos); medidas em epidemiologia (incidência, prevalência, morbidade, mortalidade); epidemiologia ebioestatística; epi- demiologia molecular; epidemiologia dos serviços de saúde; epidemiologia nutricional; fármaco epidemiologia; vigilância epidemiológica; aplicações da epidemiologia; risco; fatores de risco; probabilidade; fatores de confusão; viés e modificadores de efeito.

Foi utilizado o programa M icrosoft Excel versão 2002 para a construção da planilha eletrônica. Foram excluídos osartigos duplicados. A classificação de periódicos pela CAPES, em vigor à época da coleta dos dados, admitia três grandes categorias: periódicos internacionais e nacionais (A, B ou C). Foram identificadas as principais temáticas, as revistas mais frequentes e a distribuição das publicações no período estudado.

\section{Resultados}

A análise dos cadernos de indicadores da CAPES demonstrou que, em 2006, havia 48 programas na grandeárea de Saúde Coletiva em todo o Brasil. Embora o número total tenha duplicado em relação ao ano de $1998^{20}$, observa-se ainda uma concentração acentuada de programas na Região Sudeste (Tabela 1). A mesma concentração pode ser observada em relação à distribuição dos grupos de pesquisa: em 1997, 65,6\% deles encontrava-sena Região Sudestee, em 2006, essa concentração reduziu-se para $50,4 \%{ }^{21}$.

Deacordo com o portal daCAPES ${ }^{18}$, no período de 1998 a 2006, foram criados 25 novos programas de Saúde Coletiva, sendo que dois deles são programas específicos de epidemiologia. É interessanteobservar que, em 2007 e 2008, foram criados mais dois programas de epidemiologia, o queindica uma tendência à emergência de programas cujo recorte é o campo disciplinar. Temse, ao final do ano 2009, um total de 54 programas na área de Saúde Coletiva.

Tabela 1. Número e percentual de programas da área de Saúde Coletiva cadastrados na CAPES, por região do país.

\begin{tabular}{|c|c|c|c|c|}
\hline \multirow{2}{*}{ Região/ano } & \multicolumn{2}{|c|}{1998} & \multicolumn{2}{|c|}{2006} \\
\hline & $\mathrm{N} 0$ & $\%$ & $\mathrm{~N} 0$ & $\%$ \\
\hline Sudeste & 13 & 54,2 & 25 & 52 \\
\hline Nordeste & 07 & 29,2 & 11 & 23 \\
\hline Sul & 03 & 12,5 & 09 & 18,8 \\
\hline Norte & - & - & 02 & 4,2 \\
\hline Centro-O este & 01 & 4,2 & 01 & 2,1 \\
\hline Total & 24 & 100 & 48 & 100 \\
\hline
\end{tabular}


Com relação aos 48 programas identificados até 2006, 04 são de epidemiologia, 22 são de saúde coletiva, 09 de saúde pública, 02 de vigilância em saúde pública, 02 de saúde da mulher e da criança, 02 demeio ambiente, 01 de saúde-ambiente-trabalho, 01 de saúde da família, 01 de me dicina preventiva, 01 denutrição em saúdepública, 01 de gestão de serviços de saúde, 01 de saúde da comunidade e 01 de saúde pública baseada em evidências. Parafins desse estudo, foram analisados os 26 programas cujos cadernos de indicadores estavam disponíveis no portal CAPES, no período estudado.

A pesquisa no Brasil vem sendo impulsionada nasúltimas décadas por políticas que, efetivamente, criaram oportunidades de formação de pesquisadores em grandes centros científicos de todo o mundo e incentivaram a consolidação de programas nacionais, como comentado na introdução deste artigo. No campo da saúde pública, essa expansão tem se refletido, em especial, no crescimento da pesquisa epidemiológica ${ }^{22,23}$.

Guimarães e Gomes ${ }^{20}$ e Guimarães ${ }^{16}$, ao analisarem o diretório dos grupos cadastrados no Conselho Nacional de Desenvolvimento Científico e Tecnológico (CN Pq) em 2000, identificaram 176 grupos de pesquisa no país com pelo menos uma das suas linhas de pesquisa situada no campo da epidemiologia, o quetotalizava 320 linhas, envolvendo 813 pesquisadores, dos quais 422 eram doutores. Das 320 linhas de pesquisas epidemiológicas, 113 (64,2\%) estavam associadas à Saúde Coletiva e 15 (8,5\%), à medicina.

Com relação às linhas de pesquisa epidemiológicas, Guimarães ${ }^{24}$ agrupou-as em doze temas: acidentes, violência e trauma; bioestatística e métodos quantitativos; doenças crônicas não transmissíveis; doenças transmissíveis; epidemiologia da terceira idade; epidemiologia dos serviços; epidemiologia e ciências sociais; epidemiologia odontológica; saúde do trabalhador e epidemiologia ambiental; saúde materno-infantil, nutrição e reprodução; saúde mental e outros.

Embora o censo do CN Pq de $2008^{21}$ não disponibilize dados específicos relativos à epidemiologia, pode-se observar que a saúde foi o setor de aplicação que mais apresentou linhas de pesquisa $(25 \%)$, seguida pela educação ( $22 \%$ ). Com relação aos grupos de pesquisa segundo área de conhecimento, a Saúde Coletiva ocupou a quinta colocação, precedida pela educação, medicina, química e agronomia.

Em 2006, além da consolidação das linhas de pesquisa anteriormentemencionadas, constatouse a presença de novas linhas, como avaliação de programas, determinantes sociais, fármaco epidemiologia, desenvolvimento eaplicação demétodos estatísticos e modelagem computacional. A enumeração desses temas se mostra quase ilimitada, dado queo método epidemiológico temse revelado bastante flexível no seu diálogo com outras áreas da ciência.

No período de 2001 a 2006, os programas analisados tinham, em média, a cada ano, 248 linhas de pesquisa em Saúde Coletiva, sendo 129 em pesquisas epidemiológicas, ou seja, $52 \%$. (Gráfico 1). Esse percentual pode, em alguns programas, chegar a $100 \%$.

O padrão de crescimento da produção científica brasileira em epidemiologia não édiferente do observado para outras áreas de conhecimento no Brasil, considerando que ela tem se dado predominantemente no âmbito acadêmico, em instituições que oferecem cursos de graduação e pós-graduação. Como exemplos, podemos citar que, no ano de 2000, a USP agregava 1.356 grupos de pesquisa cadastrados no CN Pq, a UFRJ, 679 e a Fiocruz, 160. Em 2008, esses números chegam a 1.839, 822 e 296, respectivamente ${ }^{21}$.

Ciência, tecnologia eeducação qual ificada são componentes fundamentais para o desenvolvimento econômico, tecnológico e industrial dos países. Observa-se que, entre os países mais desenvolvidos, há elevada correlação entre o Produto Interno Bruto (PIB) eo desempenho científico tecnológico. Entre os dez países com maior destaque em ciência etecnologia ( $C \& T$ ), estão os Estados Unidos da América, Japão, Alemanha,

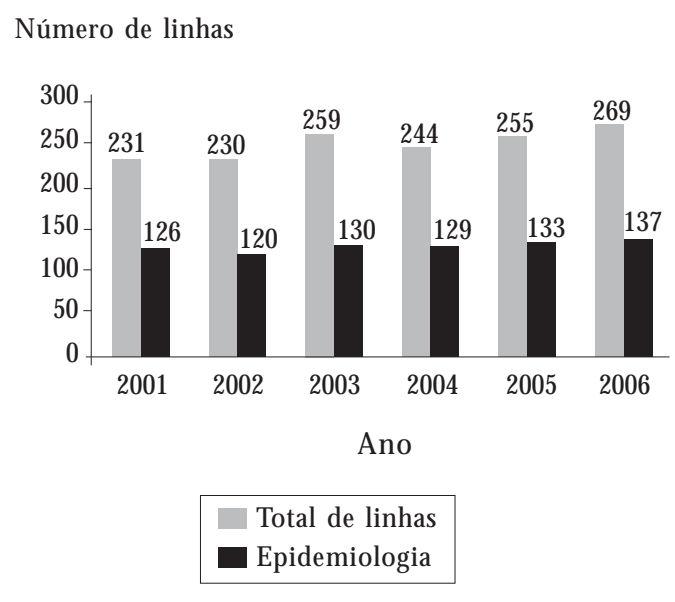

Gráfico 1. Total de linhas de pesquisa em Saúde Coletiva e total de linhas de pesquisas epidemiológicas, no período de 2001 a 2006. 
Inglaterra, França, Canadá, Itália, China, Rússia e Espanha ${ }^{16}$.

O Brasil sedistinguedentreos países em desenvolvimento por possuir um conjunto de cientistas dedicados tanto à área básica quanto às áreas de aplicação relacionadas, o que favorece a criação de soluções próprias em função de suas dimensões continentais e particularidades regionais ${ }^{25}$. Estes cientistas, entreeles muitos epidemiologistas, têm sido apoiados por um sistema de pós-graduação que forma a grande maioria dos recursos humanos especializados na área da saúde. Essas são razões suficientes para que o Brasil busque aperfeiçoar um sistema de pesquisa em saúde pactuado tanto com os serviços, como a área deinvestigação, para dar conta denovos desafios enecessidades quechegam com o século XXI.

0 aumento no número de publicações científicas nacionais se deu a partir da década de sessenta, quando a média das publicações divulgadas em periódicos indexados na base de dados do ISI (Institute for Scientific Information), era de 52 artigos científicos anuais. Em 1970, foram de apenas 64 artigos, representando $0,019 \%$ da produção mundial naqueleano. M as, em 2005, 0 Brasil foi o $15^{\circ}$ país que mais publicou no mundo ${ }^{16,24}$. Esse avanço foi reflexo da acertada decisão do governo federal, tomada nos final dos anos oitenta, para a criação do M inistério deCiência e Tecnologia, que investiu na formação de re cursos humanos, especialmente em novos doutores. Com este desempenho, o Brasil passou a contribuir com significativa parcela do total mundial dos trabalhos completos publicados em revistas de circulação internacional indexadas na ISI. Destaque para a pesquisa médica, quefoi uma das áreas mais produtivas, com crescimento médio de $19 \%$ ao ano ${ }^{24}$.

Para se compreender melhor o significado deste aumento, em 1981, o Brasil era responsável por apenas $0,44 \%$ da produção acadêmica científica mundial e ocupava a 27 a posição entre os trinta países que produziam C\&T) e, em 2001, passou a ocupar a 18a posição, sendo responsável por $1,44 \%$ da produção total mundial ${ }^{16}$.

Segundo Barros ${ }^{26}$, no período de 2001-2004, foram publicados mais de 4.500 artigos na área de Saúde Coletiva em 1.350 periódicos. Os doze mais utilizados publicaram um terço dessa produção. As revistas Cadernos de Saúde Pública, Revista de Saúde Publica e Revista Pan Americana de Salud Publica foram responsáveis por $25 \%$ do total de artigos.

Carvalho et al. ${ }^{19}$ constataram que os Cadernos deSaúde Pública ea Revista de Saúde Pública constituem os periódicos brasileiros mais antigos dedicados à Saúde Coletiva que, desde seus lançamentos, vêm sendo publicados sem interrupção. Ambos estão indexados nas principais bases bibliográficas internacionais e, juntos, lideram as estatísticas das revistas mais consultadas na base eletrônica SciELO (Scientific Electronic Library O nline; http://www.scielo.br). Ao Iongo dos anos, ambas as revistas consolidaram-se como sendo as que os pesquisadores da área de Saúde Coletiva, detentores de bolsa de produtividade em pesquisa do CN Pq, mais publicaram. Além disso, são essas revistas que publicam uma parcela expressiva da produção dos programas de pós-graduação em Saúde Coletiva no país. Os autores observaram também quea epidemiologia foi a subárea mais representada em ambos os periódicos, sendo que, na Revista de Saúde Pública, tais artigos foram, em média, responsáveis por mais de $50 \%$ do total de publicações no período de 1996 a 2003.

Previamente ao trabalho de Carvalho et al. ${ }^{19}$, Guimarães ${ }^{24}$ observou que, no período de 1996 a 1998, a revista Cadernos de Saúde Pública (CSP) já despontava como a que mais publicava artigos de cunho epidemiológico no Brasil. Foram 102 artigos, seguidos pela Revista de Saúde Pública (RSP), com 96 artigos.

No período de 2001 a 2006, as duas revistas mantiveram-senaliderança, com 375 artigos nos CSP e 242 na RSP. 0 terceiro lugar era ocupado pela Revista Ciência \& Saúde Coletiva, com 52 artigos. A produção de artigos epidemiológicos concentrava-se em revistas Qualis internacional A e B. Destacam-se revistas de prestígio internacional como Lancet, International Journal of Epidemiology, Journal of Epidemiology and Community H ealth, American Journal of Pubic $\mathrm{H}$ ealth e 0 British M edical Journal que publicaram, no período estudado, 4,5\% do total de artigos epidemiológicos produzidos por pesquisadores brasileiros.

Revistas Qualis Nacional (A, B, C) como a Revista Brasileira de Epidemiologia, o Informe Epidemiológico do SUS, somado à Revista Epidemiologia eServiços deSaúde, Cadernos deSaúde Coletiva da UFRJ e Revista de Nutrição da PUCCAM P, publicaram no período 112, 42, 39 e 25 artigos epidemiológicos.

0 volume de publicações em epidemiologia cresceu tanto na última década queatualmenteé impossível um especialista manter-se atualizado. Isso se agrava quando o público-alvo éconstituído por profissionais vinculados aos serviços de saúde. É notória a incompatibilidade entre a 
quantidade de informação produzida e a capacidade de acessar e processar em tempo hábil as informações para subsidiar políticas e intervenções baseadas em evidências. Por esta razão, foi inaugurada por revistas científicas de grande circulação uma nova estratégia, que éa de publicar artigos de revisão sistemática e meta-análises sobre os temas de maior interesse e relevância. A pesar do reconhecimento dequeas revisões sistemáticas e meta-análises constituem uma significativa fonte de informação científica, o espaço editorial destinado a este tipo de produção acadêmica ainda pode ser considerado limitado e incapaz deatender às diversas demandas, especialmente na área da saúde pública ${ }^{27}$.

Ao considerar a produção de artigos epide miológicos publicados somenteem revistas Qualis Internacional, verifica-sequerepresentam percentuais bastantesignificativosem relação ao total de artigos publicados pelos dez programas analisados (Gráfico 2). A produção total de artigos epidemiológicos representou 40\% (2.494 artigos) do total da área. Se tomarmos exclusivamente a produção internacional, podemos observar que a epidemiologia contribui, no período, com 55\% (1.826 artigos) do total.

Em relação aos objetos de estudo, observa-se que a epidemiologia abarca tanto temas tradicionais de relevância para a saúde pública, como temas contemporâneos, além de estudos específicos sobre métodos.
Silva ${ }^{5}$, ao analisar os temas apresentados no I Congresso Brasileiro de Epidemiologia, identificou que $22,3 \%$ dostrabalhosabordavam as doenças infectoparasitárias, $14,7 \%$, as desigualdades sociais, $12 \%$, a relação saúde e trabalho e $8,7 \%$, os serviços de saúde. 0 restante abordava temas como saúdematerno-infantil, vigilância epidemiológica, medicamentos, violência, entre outros.

Barreto ${ }^{22}$, ao analisar os temas dos artigos epidemiológicos publicados em revistas indexadas, no período de 1985 a 1989, constatou que $74 \%$ deleseram relacionados às doenças infecciosas ou à área materno-infantil e os restantes $26 \%$ correspondiam a artigos sobre câncer, doenças cardiovasculares e mentais, problemas ambientais enutricionais. No período de 2000 a 2004, 0 quadro inverteu-se: $40 \%$ dos artigos dedicaramsea doenças infecciosas e saúdematerno-infantil e os demais $60 \%$ dedicaram-se a outros temas, como reprodução, violência, saúde oral, entre outros.

Corroborando os achados de Barreto ${ }^{22}, 0$ presente estudo observa uma diminuição relativa de artigos voltados às doenças infecciosas e saúdematerno-infantil (33\%). Cabe ressaltar, no entanto, que os estudos voltados à aids representam grande parcela da produção sobre doenças infecciosas, o que pode ser explicado, entre outras razões, pelo incremento de recursos públicos voltados à pesquisa e ações de controle da epidemia ${ }^{28}$.

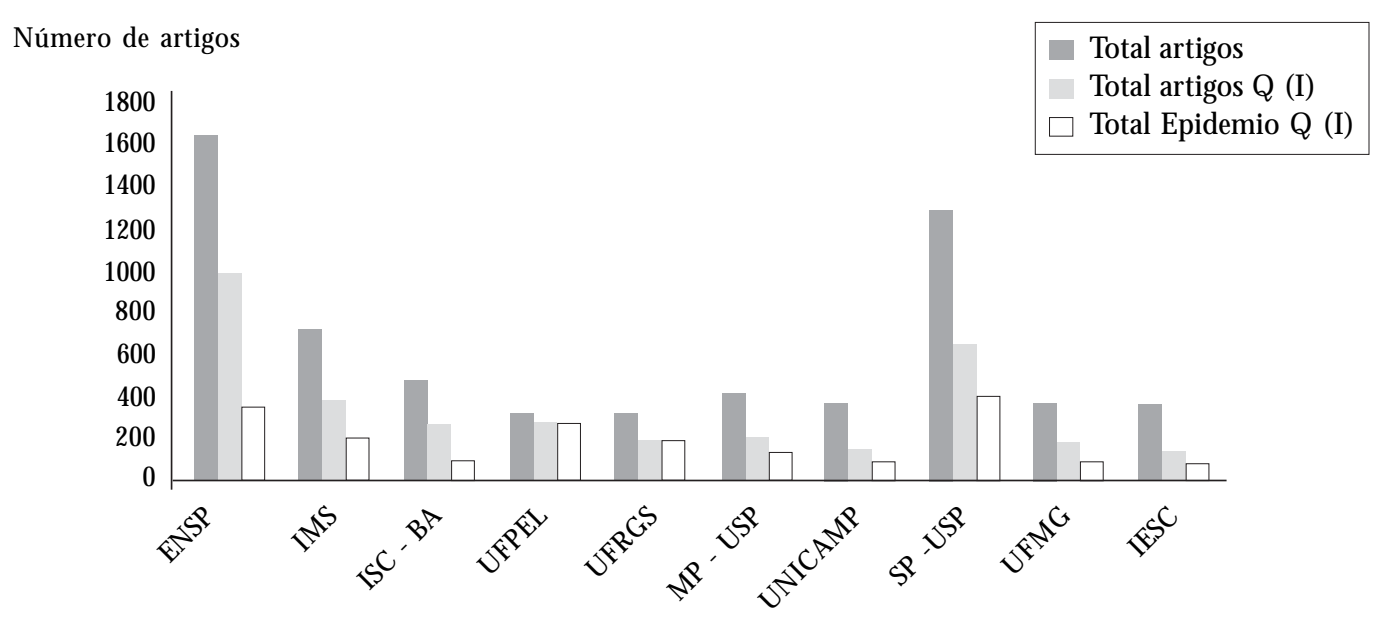

Gráfico 2. Produção de artigos de dez programas de Saúde Coletiva, no período de 2001 a 2006. 
0 Gráfico 3 apresenta os temas mais frequentes nos artigos epidemiológicos analisados no período de 2001 a 2006.

0 tema nutrição em saúde pública foi o mais prevalente entre os artigos epidemiológicos analisados (13,4\%), o que representa uma novidade em relação a períodos anteriores. A epidemia de obesidade ${ }^{29}$, aliada à permanência de altos índices de desnutriçãa ${ }^{30}$, a importância da amamentação, particularmente para a saúde de crianças que vivem em países em desenvolvimento ${ }^{31}$, a avaliação de estratégias governamentais para a redução da desnutrição ${ }^{32}$, configuram as principais questões problematizadas.

0 número de artigos voltados ao estudo da determinação social da doença também foi expressivo no período: 4,5\%, o quevai ao encontro das observações de Silva e Barros ${ }^{33}$, quando afirmam que a partir dos anos noventa, ampliou-se a preocupação de pesquisadores eagências quanto à questão das iniquidades em saúde.

Podemos observar na constituição da Saúde Pública uma tensão histórica entre explicações biológicas e sociais para a origem das doenças. As descobertas bacteriológicas propiciaram a hegemonia do modelo biológico até 0 advento da transição epidemiológica ${ }^{34}$ e 0 consequente aumento da prevalência de doenças crônicas, o que impôs à epidemiologia a busca por um novo modelo explicativo. A partir de 1990, os estudos sobre as desigualdades sociais voltam a ser privilegiados pela epidemiologia, possibilitados pelos avanços metodológicos da disciplina e impulsionados por uma visão de saúde pública queinclui, mas ultrapassa os aspectos biológicos.

É inegável a contribuição dos estudos sobre fatores de risco para a elucidação da etiologia de doenças crônicas, como as cardiovasculares ealguns tipos de câncer. No presente trabalho, esse tema éabordado em $11 \%$ dos artigos. No entanto, éimportanteconsiderar quea ênfaseem fatores de risco relacionados ao estilo de vida e às exposições ocupacionais tem levado à uma excessiva individualização do risco, o que pode enfraquecer da epidemiologia e a saúde pública. $M$ endonça ${ }^{34}$ considera que a epidemiologia molecular, a teoria dos eventos precoces na vida e a aplicação de modelos multinível podem contribuir para a elucidação de questões como as diferenças entremorbidadee mortalidade entregrupos socioeconômicos.

Em que pese a grande importância de estudos de meta-análises, principalmente no sentido de oferecer uma atual ização aos profissionais de serviço ${ }^{27}$, encontrou-se, no período estudado, um número ainda pouco expressivo de artigos ( $\mathrm{me}$ nos de $1 \%$ ). M erece destaque o aumento do número deartigos baseados em técnicas de geoprocessamento, o que está de acordo com uma crescente demanda de sua aplicação na saúde pública, especial mentena área de vigilância epidemiológica ${ }^{35}$.

Temas como saúde trabalho-ambiente, saúde mental, saúde do idoso, violência, dependên-

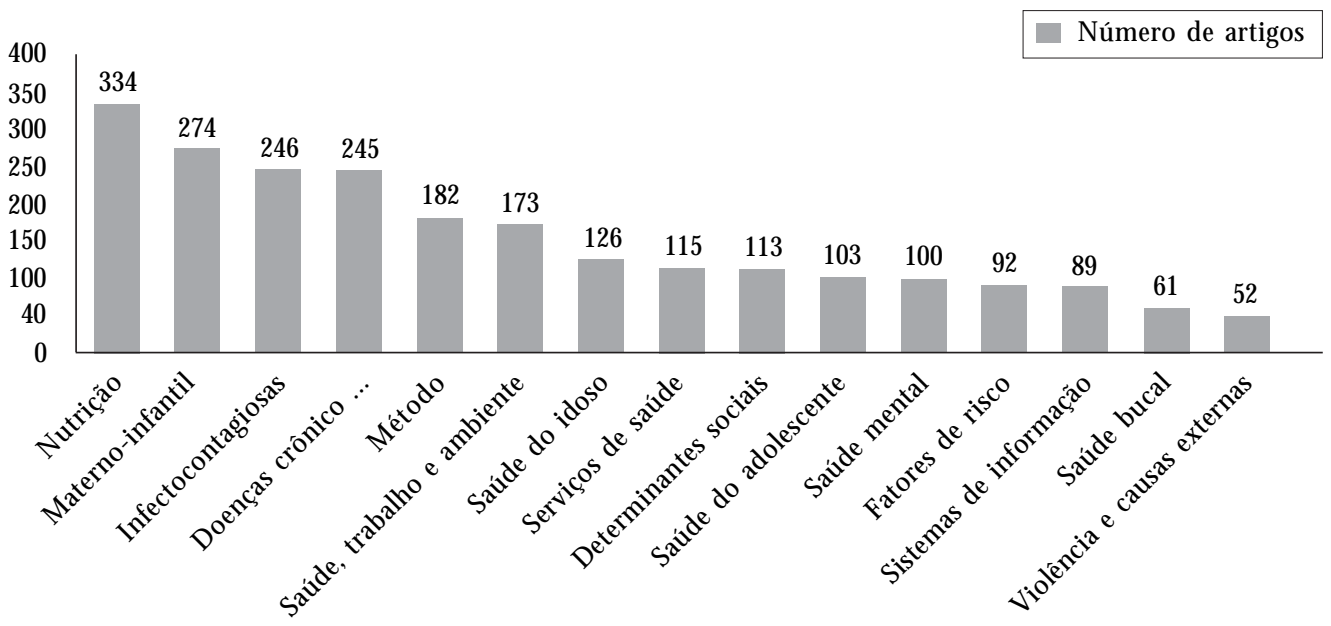

Gráfico 3. Temas mais frequentes nos artigos epidemiológicos Qualis I no período de 2001 a 2006. 
cia química (inclusive de tabaco), saúde oral e saúdeindígena têm sido problematizados expressivamente por pesquisadores da subárea.

\section{Conclusão}

A produção em epidemiologia no Brasil está vinculada, prioritariamente, a instituições acadêmicas e vem crescendo, tanto do ponto de vista quantitativo - aumento progressivo do número de artigos publicados em revistas científicas quanto do ponto de vista da incorporação de novos temas.

Observa-se, no período estudado, um aumento do número total de programas de Saúde Coletiva no país, embora ain da se mantenha uma concentração na Região Sudeste, o que se evidencia também em relação ao número de grupos de pesquisas cadastrados no CNPq.

$\mathrm{O}$ aumento na oferta de cursos de pós graduação stricto sensu em epidemiologia tem contribuído substancialmente para a formação de profissionais de saúde que atuam ou atuarão no SUS, capacitando-os a desenvolver pesquisas operacionais diretamente aplicáveis à prática profissional. Neste sentido, cabe ressaltar o grande papel que a modalidade de mestrado profissional pode desempenhar.

Verifica-se que a contribuição da epidemiologia para a produção científica da área de Saúde Coletiva é bastante expressiva. Tomando-se a produção de dez dos maiores programas de pósgraduação da área, observou-se que $40 \%$ do to- tal de artigos são epidemiológicos e, entre os artigos Qualis Internacional, a disciplina contribuiu com $55 \%$.

Com relação aos temas, nutrição em saúde pública foi o mais prevalente. Confirmou-se uma tendência de diminuição do número deartigossobre saúde materno-infantil e doenças infectocontagiosas, embora ainda ocupem o segundo lugar entre os mais abordados (destaca-se uma grande produção de artigos sobreaids). É crescente o número de artigos que abordam temas como determinantes sociais, fatores de risco, saúde-trabalhoambientee saúde do idoso. Artigos demeta-análise e geoprocessamento despontam como tendências significativas em diversos programas.

Os resultados apresentados nesteestudo permitem afirmar que, no período estudado, há tendência de aumento do volume da produção em Saúde Coletiva que, de modo geral, é expressiva na produção indexada e os temas abordados nos artigos refletiram a diversidadedeinteresses dosinvestigadores que abarcaram amplo espectro de aspectos relevantes para as políticas de saúde no país.

Conclui-se que há tendência de aumento da produção epidemiológica em publicações indexadas, abrangendo temáticas diversas e abarcando amplo espectro de aspectos relevantes para a política de saúde no país e que os sanitaristas e epidemiologistas devem continuar investindo esforços para responder às diretrizes traçadas para o desenvolvimento da área, bem como ampliar a discussão sobre temas correspondentes às prioridades definidas pelo perfil brasileiro de morbimortalidade.

\section{Colaboradores}

SRB Turci contribuiu para a concepção, pesquisa, elaboração, análise e conclusão do artigo; M CR Guilam contribuiu para a concepção, pesquisa e elaboração do artigo; MCC Câmara contribuiu para a pesquisa de dados e elaboração do artigo. 


\section{Referências}

1. Almeida TF, Vianna MIP. O Papel da epidemiologia no planejamento das ações de saúde bucal do trabalhador. Saúde soc. 2005; 14(3):144-154.

2. Barata RB. Tendências no ensino da epidemiologia no Brasil. Rev Panam Salud Publica 1997; 2(5):334341.

3. Carvalheiro JR. Processo migratório e disseminação de doenças. In: Textos de Apoio em Ciências Sociais. Rio de Janeiro: PEC/ENSP-ABRASCO; 1986.

4. Arouca ASS. 0 dilema preventivista: contribuição para a compreensão e crítica da medicina preventiva [tese]. Campinas (SP): Universidade Estadual de Campinas; 1976.

5. Silva GR. Avaliação e perspectivas da epidemiologia no Brasil. In: Anais do I Congresso Brasileiro de Epidemiologia. Rio de Janeiro: ABRASCO; 1990.

6. Silva GR. La formación de personal en función de los usos y perspectivas de la Epidemiología. Rev. bras. epidemiol. 2006; 9(1):24-31.

7. Barreto ML. Papel da Epidemiologia no desenvolvimento do Sistema Único de Saúde no Brasil: histórico, fundamentos e perspectivas. Rev. bras. epidemiol. 2002; 5(Supl 1):4-17.

8. Almeida Filho N, Castiel LD, Ayres JRCM. Riesgo: concepto básico de la epidemiología. Revista Salud Colectiva 2009; 5:323-344.

9. Ayres JRCM. Uma concepção hermenêutica de saúde. Physis 2007; 17:43-62.

10. Castiel LD, Guilam MCR, Silva PRV, Sanz J. Os riscos genômicos e a responsabilidade pessoal em saúde. Rev Panam Salud Publica 2006; 19(3):189-197.

11. Czeresnia DC. Epidemiologia: teoria e objeto. 2ª ed. Rio de Janeiro: Hucitec; 1994.

12. Czeresnia DC. Epidemiologia, Ciências Humanas e Sociais e a integração das ciências. Rev. Saude Publica 2008; 42:1112-1117.

13. Pereira GM. Epidemiologia: teoria e prática. Rio de Janeiro: Hucitec; 1999.

14. Arcoverde C. Cronologia da Associação Brasileira de Pós-Graduação em Saúde Coletiva. [acessado 2009 set 10]. Disponível em: http://www.abrasco.org.br

15. Associação Brasileira de Pós-Graduação em Saúde Coletiva. Relatório Final da I Reunião Nacional sobre Ensino e Pesquisa em Epidemiologia. 1986 [acessado 2009 set 10]. Disponível em: http://www. abrasco.org.br

16. Guimarães JA. A pesquisa médica e biomédica no Brasil. Comparações com o desempenho científico brasileiro e mundial. Cien Saude Colet 2004; 9(2):303327.

17. Barreto ML, Carmo EH. Padrões de Adoecimento e de morte da população brasileira: os renovados desafios para o Sistema Ú nico de Saúde. Cien Saude Colet 2007; 12(Supl):1779-1790.

18. Coordenação de Aperfeiçoamento de Pessoal de Nível Superior. Caderno de Indicadores 20012006.[acessado 2008 mar 26]. Disponível em: http:/ /www.capes.gov.br

19. Carvalho L, Coimbra C, Souza-Santos R, Santos RV. Produção e citação em Saúde Coletiva: um olhar a partir dos periódicos dos Cadernos de Saúde Pública e Revista de Saúde Pública. Cad Saude Publica 2007; 23(12):3023-3030.
20. Guimarães JA, Gomes J. Duas décadas de desempenho científico excepcional na América Latina. In: Trossero AP. Los laberintos del futuro. Santa Fé: Universidade del Litoral; 2001. p. 69-90.

21. Conselho Nacional de Pesquisa. Censo CN Pq 2008. [acessado 2009 fev 04]. Disponível em: http://dgp. cnpq.br/censos/series_historicas/index_grupos.htm

22. Barreto $M L$. Crescimento e tendência da produção científica em epidemiologia no Brasil. Rev. Saude Publica 2006; 40(N Esp):79-85.

23. Barata RB. Epidemiologia Social. Rev. bras. epidemiol. 2005; 8(1):7-17.

24. Guimarães R, Lourenço $R$, Cosac $S$. A pesquisa em epidemiologia no Brasil. Rev. Saude Publica 2001; 35:321-340.

25. Diniz CWP. Os dilemas do desenvolvimento científico e tecnológico brasileiro. Cien Saude Colet 2004; 9(2):271-274.

26. Barros AJD. Produção científica em saúde coletiva: perfil dos periódicos e avaliação pela Capes. Rev. Saude Publica 2006; 40(n spe):43-49.

27. Vettore MV, Bastos FI. Revisões em epidemiologia: linhas de estudos correntes e perspectivas futuras. Cad Saude Publica 2008; 24(suppl. 4):S472-S473.

28. Fonseca EM, Nunn A, Souza-Junior PB, Bastos FI, Ribeiro JM. Descentralização, AIDS e redução de danos: a implementação de políticas públicas no Rio de Janeiro, Brasil. Cad Saude Publica [periódico na Internet] 2007 [acessado 2010 fev 10]; 23(9): [cerca de 11 p.]. Disponível em: http://www.scielo. $\mathrm{br} / \mathrm{pdf} / \mathrm{csp} / \mathrm{v} 23 \mathrm{n} 9 / 14 . \mathrm{pdf}$

29. Sichieri R. Dietary patterns and their association with obesity in the Brazilian city of Rio de Janeiro. Obesity Research 2001; 10(1):42-48.

30. Batista Filho M, Rissin A. A transição nutricional no Brasil: tendências regionais e temporais. $C$ ad Saude Publica [periódico na Internet] 2003; 19(suppl.1):[cerca de 11 p.]. Disponível em: http:/ /www.scielosp.org/pdf/csp/v19s1/a19v19s1.pdf

31. Lauer JA, Betrán AP, Barros AJ, de Onís M. Deaths and years of life lost due to suboptimal breast-feeding among children in the developing world: a global ecological risk assessment. Public $\mathrm{H}$ ealth $\mathrm{N}$ utr. 2006; 9(6):673-683.

32. Paes-Sousa R. Pobreza e desnutrição: uma análise do Programa Fome Zero sob uma perspectiva epidemiológica. Saúde soc. 2003; 12(1):21-30.

33. Silva JBS, Barros MBA. Epidemiologia e desigualdade: notas sobre a teoria e história. Rev Panam Salud Publica 2002; 12(6)375-383.

34. M endonça GAS. Tendências da investigação epidemiológica em doenças crônicas. Cad Saude Publica 2001; 17(3):697-703.

Artigo apresentado em 09/03/2010

Aprovado em 19/04/2010

Versão final apresentado em 19/04/2010 